\title{
Habilidades Motrices en Niños de 6 a 13 Años del Colegio Meira Del Mar de Barranquilla
}

\section{Motor Skills in Children 6 to 13 Years of Meira Del Mar School from Barranquilla}

\author{
Eulalia Amador Rodero ${ }^{1}$ \\ Universidad Libre
}

Mónica Gómez Barbosa ${ }^{2}$

Universidad Simón Bolívar

Leslie Montealegre Esmeral ${ }^{3}$

Universidad Libre

\section{RESUMEN}

Introducción: El movimiento es la primera forma de comunicación que tiene el ser humano con el medio. Aparece en el momento de la gestación y se va perfeccionando durante los primeros años de vida. Objetivo: Identificar las habilidades motoras gruesas y finas de los niños de 6 a 13 años del colegio Meira Del Mar del Distrito de Barranquilla. Materiales y métodos: Estudio descriptivo transversal en 120 escolares. La técnica para la recolección de información fue la Batería de Oseretsky, que permitió efectuar un análisis sobre los componentes de la motricidad en los niños. Resultados: Los niños presentaron un mejor desempeño que las niñas al realizar movimientos simultáneos (38\%). En cuanto a la edad, se observó que los escolares de 8 a 10 años tuvieron mejor desempeño en la coordinación estática positiva (67\%), seguido por la ausencia de sincinesis (58\%) y la coordinación dinámica de las manos (57\%). Conclusión: Las habilidades motoras maduran a medida que el niño avanza en edad. El desempeño de las habilidades motoras en los niños y niñas estudiados fue bueno; pero los niños tuvieron más habilidad en comparación con las niñas.

Palabras Clave: habilidades motrices, fisioterapia, motricidad, evaluación motora (Fuente: DeCS).

\begin{abstract}
Introduction: Movement is the first form of communication that human beings have with the environment, appears at the time of pregnancy and gradually perfected during the first years of life. Objective: Identify gross and fine motor skills of children aged 6 to 13 of the College Meira Del Mar College from Barranquilla. Materials and methods: Cross-sectional descriptive study in 120 school children. The technique for the collection of information was the Oseretsky battery, allowing an analysis on the components of the motility in children. Results: children presented a better performance than the girls to perform simultaneous movements (38\%), in terms of the age was observed that school children 8 to 10 years had better performance in coordination static positive (67\%), followed by the absence of synkinesis (58\%) and dynamic coordination of the hands (57\%). Conclusion: motor skills become mature measures child advancing in age. The performance of motor skills in the children studied was good; the children had more skills in comparison with girls.
\end{abstract}

Keywords: motor skills, physical motor, motor assessment (Source: MeSH, NLM).

Fisioterapeuta. Especialista en Educación con Énfasis en Evaluación Educativa. Especialista en Gerencia de Servicios de Salud. Magíster en Neurociencias con énfasis en Trastornos por Déficit de Atención e Hiperactividad. Correo electrónico: eamador62@ hotmail.com

2 Fisioterapeuta. Especialista en Epidemiología. Especialista en Gestión de Proyectos Educativos. Magíster en Educación con Énfasis en Cognición. Correo electrónico: monigomez0311@ hotmail.com

3 Fisioterapeuta. Magister en Salud Pública. Correo electrónico: lesliepiedad@gmail.com 


\section{Introducción}

El desarrollo motor o adquisición de las habilidades motoras gruesas y finas constituye la manifestación externa de la madurez progresiva del sistema nervioso central. La proliferación de las dendritas y la mielinización de los axones son los responsables fisiológicos de los progresos observados en el niño. Pero, para que estos progresos se den, además de la integridad de los sistemas biológicos, se necesita un medio ambiente que lo favorezca, el cual, a su vez, depende de los contextos, culturas y regiones geográficas. Por consiguiente, las características concretas de esas habilidades estarán determinadas por el medio en el cual se desenvuelve el individuo (1-3).

Gessel (4) caracterizó el desarrollo motor y los comportamientos motores tomando como base la neuromaduración de la corteza cerebral. Higgins (5), por su parte, afirmó que el ritmo del proceso de maduración es afectado por la cantidad y el tipo de experiencias de aprendizaje, y éstas por el ambiente sociocultural.

Más recientemente, se viene investigando sobre la forma como la teoría de los sistemas dinámicos se ha aplicado a las ciencias del desarrollo (6). Este modelo se inspiró en los trabajos de Bernstein, y desde él se asume a la conducta motriz como la interacción de muchos subsistemas en un contexto y tarea específica.

Ahora bien, las características del desarrollo motor pueden determinarse mediante una evaluación sistemática. En este sentido, el fisioterapeuta cuenta con las herramientas y competencias para hacerlo de manera confiable y, a partir de los hallazgos, puede formular un plan de intervención é interacción (7). Dicha evaluación motora se fundamenta en una serie de elementos visibles que permiten detectar, a través del desempeño motriz, la interrelación entre lo perceptual, lo cognitivo, lo afectivo y lo psicomotor, así como identificar la manera en que esa interrelación interfiere el logro de los desempeños motores (8).

Más concretamente, en cuanto a la evaluación del desempeño motor en los niños, existen estudios que permiten caracterizar poblaciones estudiadas. Entre estos se encuentran: los trabajos de Derri y Pachta, que se centran enel efecto de dos estilos de enseñanza, mediante el descubrimiento guiado y el uso del test de Desarrollo Motor Grueso (9); el de Torres (10), quien aplicó la Batería Eurofit a niños con altas capacidades intelectuales; y la evaluación basada en la utilización de la Batería Test Motor de Oseretsky (11). Otros estudios han demostrado que las dificultades en el desempeño motor pueden identificarse tempranamente (12), y se recomienda que al momento de seleccionar pruebas para la evaluación se tomen en cuenta la validez y confiabilidadde los mismos (13).

En Colombia, pocos estudios han abordado la evaluación del desarrollo motor. Hormiga (14), y Amar $(15,16)$ lo hicieron mediante la aplicación de la Escala Abreviada del Desarrollo, la cual evalúa el desarrollo mental y motor en niños y niñas hasta los cinco años de edad, aunque no evalúa de manera integral las habilidades motrices. $Y$ en el Caribe colombiano, la pesquisa realizada de nuestra parte no evidenció trabajos que estudien el desarrollo motor bajo el enfoque fisioterapéutico.

Nuestro objetivo de estudio consistió en identificar las habilidades motoras y gruesas de los niños de 6 a 13 años del Colegio Meira del Mar del Distrito de Barranquilla.

\section{Materiales y Métodos}

Se trata de un estudio descriptivo transversal. La población estuvo conformada por 175 escolares de 6 a 13 años matriculados en el Colegio Meira del Mar del Distrito de Barranquilla. De estos niños, 120 cumplieron los siguiente criterios de inclusión: un rango de edad de 6 a 13 años; el no padecer alteraciones $\mathrm{y} / \mathrm{o}$ limitaciones físicas, mentales $\mathrm{y} / \mathrm{o}$ sensoriales; y firma de consentimiento por parte de los padres o acudientes.

Se hizo la evaluación de las siguientes habilidades motrices: coordinación estática, que se define como el equilibrio entre las acciones de los grupos musculares antagonistas, ésta se establece en función del tono y permite la conservación voluntaria de las actitudes; coordinación dinámica de las manos, la cual hace referencia al ajuste corporal necesario para afrontar las demandas motrices que exigen el uso particular de algún segmento corporal; coordinación dinámica general, explicada como una acción en que intervienen gran cantidad de segmentos musculares, ya sean de extremidad superior, inferior o ambas a la vez; rapidez de movimientos, definida como la capacidad de realizar los movimientos con máxima velocidad en relación con el tiempo empleado; movimientos 
simultáneos, son movimientos coordinados que resultan de la acción cerebelosa, se caracterizan por la integración y equilibrio entre músculos agonistas y antagonistas; y ausencia de sincinesis, determinada como las reacciones parásitas de imitación de los movimientos contralaterales y de movimientos peribucales y linguales. Todo ello se hizomediante la aplicación de la batería de Oseretsky, la cual permite un análisis de los distintos componentes de la motricidad (17). En cuanto a las variables sociodemográficas, se tuvo en cuenta la edad y sexo.

El análisis y procesamiento de los resultados se realizó mediante el software Statgraphics Plus, consolidándose en tablas de frecuencias multivariables.

\section{Resultados}

Lo primero a destacar es que los niños presentaron los mejores desempeños y que en ambos sexos hubo un bajo desempeño en lo concerniente a la rapidez de movimiento. Además, la coordinación estática fue la habilidad con mejor desempeño (49\%); seguida de la coordinación dinámica de las manos $(47 \%)$ y de la coordinación dinámica general (43\%).

Con relación a los movimientos simultáneos, el 38\% de los niños y el $32 \%$ de las niñas no superaron la prueba, de acuerdo con los criterios de evaluación establecidos en la Batería (Tabla 1).

Tabla 1. Distribución de las habilidades motoras según el sexo en los niños encuestados

\begin{tabular}{lcccc}
\hline \multirow{2}{*}{ HABILIDADES MOTRICES } & \multicolumn{2}{c}{ FEMENINO } & \multicolumn{2}{c}{ MASCULINO } \\
\cline { 2 - 5 } & Presente & Ausente & Presente & Ausente \\
\hline Coordinación estática & $47(39 \%)$ & $7(6 \%)$ & $59(49 \%)$ & $7(6 \%)$ \\
Coordinación dinámica de las manos & $32(27 \%)$ & $22(18 \%)$ & $56(47 \%)$ & $10(8 \%)$ \\
Coordinación dinámica general & $38(32 \%)$ & $16(13 \%)$ & $51(43 \%)$ & $15(12 \%)$ \\
Rapidez de movimientos & $30(25 \%)$ & $24(20 \%)$ & $32(27 \%)$ & $34(28 \%)$ \\
Movimientos simultáneos & $16(13 \%)$ & $38(32 \%)$ & $20(17 \%)$ & $46(38 \%)$ \\
Ausencia de sincinesis & $36(30 \%)$ & $18(15 \%)$ & $54(45 \%)$ & $12(10 \%)$ \\
\hline
\end{tabular}

La Tabla 2 muestra los resultados de las habilidades motrices de acuerdo con el rango de edad. En este caso, hubo mejor desempeño en los escolares de 8 a 10 años, presentándose con mayor frecuencia (67\%) la coordinación estática positiva, seguida por la ausencia de sincinesis $(58 \%)$ y la coordinación dinámica de las manos $(57 \%)$.

Por otra parte, dentro de los resultados, llama la atención que el $55 \%$ de los escolares de 8 a 10 años evaluados no superaron la prueba para los movimientos simultáneos. En el grupo de escolares de 6 a 7 años, se observó al respecto un bajo desempeño comparado con el grupo de 8 a 10 años. En cambio, hubo mejor desempeño (positivo) en la coordinación estática (18\%), la coordinación dinámica general $(17 \%)$ y la ausencia de sincinesis (13\%). El grupo de 11 a 13 años no superó la prueba para movimientos simultáneos $(7 \%)$ y rapidez de movimiento $(5 \%)$. Sin embargo, es notoria la escasez del número de los niños que conformaron este grupo etario.

Tabla 2. Distribución de frecuencia de las habilidades motoras según la edad de niños y niñas sujetos de estudio

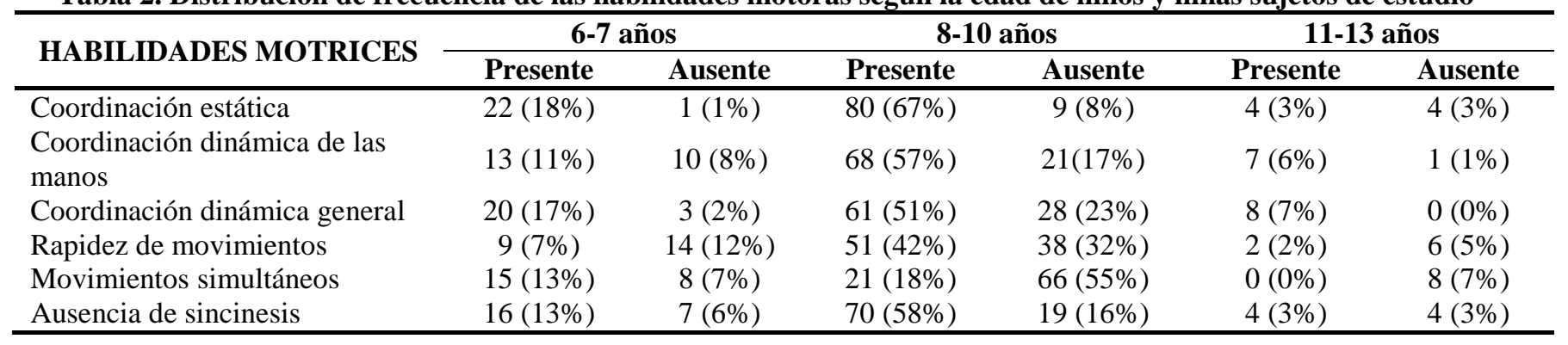

Cienc. innov. salud. Junio 2013; 1 (1): 4 - 10. Universidad Simón Bolívar (Col). ISSN: 2344-8636 http://portal.unisimonbolivar.edu.co:82/rdigital/innovacionsalud 
En el análisis de respuestas positivas de los escolares por las estructuras de edad y sexo, se observó que, en el grupo de 6 a 7 años, las niñas superaron a los niños en las pruebas, excepto en la coordinación dinámica general, en la cual las respuestas de ambos sexos fue similar (8\%). En los escolares de 8 a 10 años y de 11 a 13 años, el comportamiento fue diferente: en ambos grupos, los niños superaron a las niñas en todas las habilidades motoras evaluadas (Tabla 3 ).

En cuanto a los resultados negativos, en el grupo de 6 a 7 años, se observó que el $22 \%$ de las niñas no superó las pruebas para habilidades motoras, a diferencia de los niños, quienes mostraron respuesta negativa en un 13\%. En el intervalo de 8 a 10 años, los escolares no tuvieron éxito al realizar las pruebas para coordinación dinámica general, rapidez de movimientos y movimientos simultáneos, siendo éste último el de mayor peso porcentual (73\%) para ambos sexos. En el grupo de 11 a 13 años, el 7\% de los escolares fracasaron al realizar la prueba para movimientos simultáneos. En este intervalo de edad, se observa que existe una menor proporción de niños con respuesta negativa al realizar las pruebas $(20 \%)$.
Al analizar las frecuencias de superación de las actividades evaluadas mediante la batería de Oseretsky, se observó que el $90 \%$ de los escolares superaron las pruebas para habilidad motora, lo cual indica un buen desempeño de la motricidad en los niños y niñas estudiados.

Al desagregar estos resultados por grupos etarios y sexo, encontramos que los niños de 6 años lograron un $90 \%$ de superación, mientras que las niñas de la misma edad alcanzaron el 37\%, con una diferencia porcentual entre ambos sexos del 53\%. Para el grupo de 7 años, las niñas alcanzaron a superar el test en un $80 \%$ y los niños en un $75 \%$, con una diferencia porcentual de 0.5 . Los escolares de 8 a 10 y de 11 a 13 años mostraron un comportamiento similar en la superación de pruebas, pues, la diferencia entre ambos sexos fue de $0.3 \%$.

De acuerdo con las diferencias porcentuales existentes entre los grupo etarios y después de haber realizado el análisis estadístico correspondiente, se observó que el grupo de niños de 6 años es el que presenta mayor desviación $(29,4 \%)$. A partir de los 7 años, este tipo de diferencias disminuye, alcanzando mayor homogeneidad entre los grupos. Para los niños de 7 años, la desviación típica fue de 2,3, y para los de 8 a 13 años, de 1,4 .

Tabla 3. Distribución de las habilidades motoras según sexo y rango de edad

\begin{tabular}{|c|c|c|c|c|c|}
\hline \multirow{2}{*}{ HABILIDADES MOTRICES } & \multirow{2}{*}{ Genero } & \multirow{2}{*}{ Presencia / Ausencia } & \multicolumn{3}{|c|}{ Rango de edad } \\
\hline & & & 6-7 años & 8 -10 años & 11-13 años \\
\hline \multirow{3}{*}{ Coordinación estática } & Niños & $\mathrm{P}$ & $1(08)$ & $45(37)$ & $4(3)$ \\
\hline & \multirow{2}{*}{ Niñas } & $\mathrm{A}$ & $0(0)$ & $5(4)$ & $2(2)$ \\
\hline & & $\mathrm{P}$ & $12(10)$ & $35(29)$ & $0(0)$ \\
\hline \multirow{5}{*}{ Coordinación dinámica de las manos } & \multirow{2}{*}{ Niños } & $\mathrm{A}$ & $1(1)$ & $4(3)$ & $2(3)$ \\
\hline & & $\mathrm{P}$ & $6(5)$ & $44(37)$ & $6(5)$ \\
\hline & \multirow{3}{*}{ Niñas } & $\bar{A}$ & $4(3)$ & $6(5)$ & $0(0)$ \\
\hline & & $\mathrm{P}$ & $7(6)$ & $24(20)$ & $1(1)$ \\
\hline & & $\mathrm{A}$ & $6(5)$ & $15(12)$ & $1(1)$ \\
\hline \multirow{4}{*}{ Coordinación dinámica general } & \multirow{2}{*}{ Niños } & $\mathrm{P}$ & $10(8)$ & $35(29)$ & $6(5)$ \\
\hline & & A & $0(0)$ & $15(12)$ & $0(0)$ \\
\hline & \multirow{2}{*}{ Niñas } & $\mathrm{P}$ & $10(8)$ & $26(22)$ & $2(2)$ \\
\hline & & $\mathrm{A}$ & $3(2)$ & $13(11)$ & $0(0)$ \\
\hline \multirow{4}{*}{ Rapidez de movimientos } & \multirow{2}{*}{ Niños } & $\mathrm{P}$ & $4(3)$ & $27(22)$ & $1(1)$ \\
\hline & & A & $6(5)$ & $23(19)$ & $5(4)$ \\
\hline & \multirow{2}{*}{ Niñas } & $\mathrm{P}$ & $5(4)$ & $24(20)$ & $1(1)$ \\
\hline & & $\mathrm{A}$ & $8(7)$ & $15(12)$ & $1(1)$ \\
\hline \multirow{4}{*}{ Movimientos simultáneos } & \multirow{2}{*}{ Niños } & $\bar{P}$ & $5(4)$ & $15(12)$ & $0(0)$ \\
\hline & & A & $5(4)$ & $35(29)$ & $6(5)$ \\
\hline & \multirow{2}{*}{ Niñas } & $\mathrm{P}$ & $10(8)$ & $6(5)$ & $0(0)$ \\
\hline & & A & $3(2)$ & $33(44)$ & $2(2)$ \\
\hline \multirow{4}{*}{ Ausencia de sincinesis } & \multirow{2}{*}{ Niños } & $\mathrm{P}$ & $9(7)$ & $42(35)$ & $3(2)$ \\
\hline & & A & $1(1)$ & $8(7)$ & $3(2)$ \\
\hline & \multirow{2}{*}{ Niñas } & $\overline{\mathrm{P}}$ & $7(6)$ & $28(23)$ & $1(1)$ \\
\hline & & A & $6(5)$ & $11(9)$ & $1(1)$ \\
\hline
\end{tabular}

$\mathrm{P}=$ Presente $\mathrm{A}=$ Ausente

Cienc. innov. salud. Junio 2013; 1 (1): 4 - 10. Universidad Simón Bolívar (Col). ISSN: 2344-8636

http://portal.unisimonbolivar.edu.co:82/rdigital/innovacionsalud 


\section{Discusión}

En general, la evaluación de las habilidades motoras arrojó mejor desempeño en los niños en comparación con las niñas. Esto podría obedecer a lo planteado por Easton, Enns y Rowland, citados por Rodríguez (18), en relación a que las mujeres han sido tradicionalmente menos orientadas hacia la realización de actividades físicas, por lo que es posible que las diferencias observadas a favor de los niños en las diversas investigaciones se deban a factores de tipo sociocultural que limitan la ejecución en niñas. La falta de modelos apropiados, la conducta de padres y compañeras y las barreras psicológicas, tales como identidad de roles sexuales y percepción de falta de competencia, pueden inhibir la realización de actividad física en las chicas, lo cual se agrava por la actual tendencia hacia el uso de los juegos interactivos, que constituyen factores predisponentes a la inactividad física.

En cuanto a la presentación de las habilidades motoras según la edad, el grupo de escolares en el cual se observó mayor peso porcentual fue el de 8 a 10 años. Resultados estos que son consistentes con los encontrados por Rodríguez en niños brasileros (18). Al respecto, Cratty, citado por Rodríguez (18), afirma que la edad escolar abarca un período comprendido entre los 6-7 años y los 12, y que esta edad resulta de gran importancia para un adecuado desarrollo motor y para la adquisición de habilidades motrices nuevas. Este período supone en general la transición de habilidades motrices fundamentales refinadas hasta el inicio y posterior establecimiento de los primeros juegos y habilidades deportivas.

El desempeño motor entre los 5 y 18 años se caracteriza por un desarrollo progresivo, variado e individual (19), lo cual se interpreta como una progresión en la adquisición y refinamiento de las habilidades y destrezas motoras, que son una señal de la maduración neurológica en la escala evolutiva del ser humano (20). En el presente estudio, se observó un mejor desempeño a medida que los niños y niñas progresaban en edad cronológica. Ello se explica por el hecho de que el desarrollo de la destreza guarda relación con el desarrollo típico del cerebro y de la fuerza muscular (21). Además, la edad y la velocidad de movimiento en las manos se asocian con la capacidad de cruzar la línea media. En general, puede decirse sobre lo anterior que hay una correlación directamente proporcional entre destreza manual y desarrollo neurológico (21).

En el grupo de 6 a 7 años, las niñas superaron a los niños en las pruebas de evaluación, mientras que en los escolares de 8 a 13 años, los niños superaron a las niñas. Esto confirma los estudios de Rodríguez (18), quien propone que aunque se observan diferencias significativas en función de la edad y el sexo, resulta evidente que la edad se relaciona con la ejecución motora en mayor medida que el sexo. Así, en general, los cambios progresivos con la edad tienen un carácter bastante lineal.

En lo que se refiere al sexo, Ruiz (22) y Junaid (23) han establecido comparaciones pertinentes, y los resultados han sido rotundos: las niñas superan a los niños en actividades manuales y equilibrio, mientras que los niños superan a las niñas en actividades que involucran la coordinación visomotora, como por ejemplo, juego de pelota. También Sigmundsson observó que el desempeño de los niños en actividades que involucran destreza manual y equilibrio se encontraba significativamente por debajo con respecto al de las niñas (24).

En este último sentido, Ruiz (25) demostró que las diferencias en el desempeño motor se da en dos dimensiones: competencias deportivas perceptivas y condición física deportiva. Las niñas presentan valores más bajos en aspectos relacionados con la competencia física y deportiva, lo cual a su vez coincide con lo propuesto por Larson (26), en relación a que el desarrollo motor parece seguir un curso diferente en niñas y en niños. Estos aspectos deben tenerse en cuenta para la evaluación clínica de la función motora en los niños.

Además, se ha observado que los niños pueden tener un desarrollo del control postural más tardío con respecto a las niñas (27). La destreza y la fuerza en las habilidades motoras caracterizan a los niños mientras que el equilibrio estático y la coordinación caracterizan a las niñas (28).

En el presente estudio se observó que en su presentación las habilidades motoras fueron heterogéneas en los escolares de 6 años, con una diferencia porcentual entre ambos sexos del 53\% y desviación típica de 29,4. A partir de los 7 años, estas

Cienc. innov. salud. Junio 2013; 1 (1): 4 - 10. Universidad Simón Bolívar (Col). ISSN: 2344-8636 
diferencias disminuyen y la presentación de las habilidades motoras son homogéneas en la población, mostrando diferencias porcentuales que no superan el $0.5 \%$ y una desviación estándar entre 2,3 a 1,4 . La edad y el crecimiento son factores determinantes en el desarrollo de las habilidades motoras (28), por ello, Rodríguez (18) expresa que la edad prima sobre el sexo en el desarrollo motriz, siendo ésta un factor determinante de la mejoría en la ejecución motora.

Se concluye que la madurez neurológica, el desarrollo de la fuerza muscular y la destreza son factores determinantes en la progresión de las habilidades motoras y van apareciendo a medidas que el niño avanza en edad, de manera gradual, organizada y coordinada. En general, el desempeño de las habilidades motoras en los niños y niñas del colegio Meira Del Mar del Distrito de Barranquilla fue bueno. Los niños tuvieron más habilidad en cada una de las pruebas en comparación con las niñas, pero las diferencias en el sexo no fueron tan determinantes como la edad.

El desempeño de las habilidades motoras mejoró progresivamente en relación a la edad, exceptuando los movimientos simultáneos. Esto se corresponde con el proceso evolutivo del niño, ya que, a medida que aumenta la edad cronológica, el sistema nervioso optimiza sus conexiones neuronales y se da una mayor madurez cerebral. Todo ello se evidencia en los resultados, al observarse que, a partir de los 8 años, las diferencias porcentuales disminuyen.

Por último, las dificultades presentadas durante las pruebas por los 120 niños participantes son comunes en los diversos grupos etarios, entre ellas, movimientos simultáneos y rapidez de movimientos. Esto sugiere la revisión de las subpruebas mencionadas para determinar si son sensibles y válidas para esta población.

Por todo lo dicho, entonces, los resultados del estudio generan la necesidad de seguir investigando para crear, diseñar y validar baterías propias de la profesión, que evalúen las habilidades motrices desde la perspectiva de Calidad del Movimiento Corporal Humano.

\section{Referencias}

1. Paraskevi G, Kyparos A, Fotiadou E, Angeloupoulou N. The effect of residence area and mother's education on motor development of preschool aged children in Greece. Early Child Development and Care. 2007; 177 (5): 479-492.

2. Venetsanou F, Kambas A. Environmental factors affecting preschoolers' motor development. Early Childhood Educ J. 2010; 37:319-327.

3. BerguerS, Theuring $\mathrm{C}$, Adolph K. How and when infants learn to climb stairs. Infant Behav Dev. 2007; 30 (1): 36-49.

4. Gessell A, Ilg FL, Ames LB. Infant and child in the culture of today. New York: Harper \& Row; 1974.

5. Higgins S. Motor skill acquisition. Phys Ther. 1991; 71 (2): 123-139.

6. Fogel A. Theoretical and applied Dynamic Systems research in developmental science. Child Development Perspectives. 2011; 0 (0): 1-6.

7. Kenyon LK, Blackinton MT. Applying motorcontrol theory to physical therapy practice: a case report. Physiother Can. 2011; 63(3): 345-354.

8. Morrow JR. Measurement and evaluation in human performance. 2nd ed. USA: Human Kinetics; 2000.

9. Derri V, Pachta M. Motor skills and concepts acquisition and retention: a comparison between two styles of teaching. Revista Internacional de Ciencias del Deporte. 2007; 9(3); 37-47.

10. Torres GA, Sanz AB, Ochando GC. Aplicación de la Batería EUROFIT a niños con altas capacidades intelectuales. Disponible en Internet en

http://www.cienciadeporte.com/congreso/04\%20v al/pdf/c27.pdf. Diciembre 11 de 2009.

11. Rosa J, Márquez S.Evaluación de la ejecución motora en la edad escolar mediante los tests motores de Lincoln-Oseretsky. Revista Motricidad. 1996; 2: 129-147.

12. Bond C, Cole M, Crook H, Fletcher J, Lucanz J, Noble J. The development of the Manchester Motor Skills Assessment (MMSA): An initial evaluation. Educational Psychology in Practice. 2007; 23 (4): 363-379.

13. Wiart L, Darrah J. Review of four tests of gross motor development. Developmental Medicine \&Child Neurology. 2001; 43: 279-285.

14. Hormiga C, Camargo D, Orozco L. Reproductibilidad y validez convergente de la

Cienc. innov. salud. Junio 2013; 1 (1): 4 - 10. Universidad Simón Bolívar (Col). ISSN: 2344-8636

http://portal.unisimonbolivar.edu.co:82/rdigital/innovacionsalud 
Escala Abreviada Del Desarrollo y una traducción al español del instrumento Neurosensory Motor Development Assessment. Revista Biomédica. 2008; 28:327-346.

15. Amar J, Tirado D. Promoviendo la inclusión social en los primeros años. Salud Uninorte. 2007; 23 (2): 162-171.

16. Amar J, Abello R, Tirado D. Efectos de un programa de atención integral a la infancia en el desarrollo de niños de sectores pobres en Colombia. Revista Investigación y Desarrollo. 2005; 13 (1): 60-77.

17. Manual Test de Desarrollo Motor de Ozeretzky. Madrid: Psymtéc.

18. Rodríguez C, Cabral A, Rodríguez L, Márquez S. Evaluación de la ejecución motora en niños brasileros en edad escolar. Apunts Educación Física y Deportes. 2007; 89: 31-39

19. Largo RH, Caflish JA, Hug F, Muggli K, Molnar AA, Molinari L, et al. Neuromotor development from 5 to 18 years. Part 1: timed performance. Dev Med Child Neurol. 2001; 43(7):436-443.

20. De Bellis M, Keshavan MS, Beers S, Hall J, Frustaci K, Masalehdan A, et al. Sex differences in brain maturation during childhood and adolescence. Cerebral Cortex. 2001; 11: 552-557.

21. Hill EL, Khanem F. The development of hand preference in children: the effect of task demands and links with manual dexterity. Brain and Cognition. 2009; 71 (2): 99-107.

22. Ruiz PL, Graupera SJ. Un estudio transcultural de la competencia motriz en escolares de 7 a 10 años: utilidad de la Batería Movement ABC. Revista Española de Pedagogía. 2005; 63 (231): 289-308.

23. Junaid K, Fellowes S. Gender Differences in the Attainment of Motor Skills on the Movement assessment Battery for Children. Phys Occup Ther Pediatr. 2006; 26(1/2): 5-11.

24. Sigmundsson H, Stølan M. Motor Development: exploring the motor competence of 4-year-old Norwegian children. Scandinavian Journal of Educational Research. 2003; 47 (4): 451-459.

25. Ruiz LM. Problemas evolutivos de coordinación motriz y autoconcepto físico en escolares de educación primaria. Estudios de Psicología. 2008; 29 (2): 163-172.

26. Larson JC, Mostofsky SH, Goldberg MC, Cutting LE, Denckla MB, Mahone EM.Effects of Gender and Age on Motor Exam in Typically Developing
Children. DevNeuropsychol. 2007; 32(1): 543562.

27. Nolan L, Grigorenko A, Thorstensson A. Balance control: sex and age differences in 9- to 16-yearolds. Dev Med Child Neurol. 2005; 47 (7): 449454.

28. Shala M. Assessing gross motor skills of Kosovar preschool children. Early Child Dev Care. 2009; 179 (7): 969-976.

Cienc. innov. salud. Junio 2013; 1 (1): 4 - 10. Universidad Simón Bolívar (Col). ISSN: 2344-8636 http://portal.unisimonbolivar.edu.co:82/rdigital/innovacionsalud 